Voix et Images

\title{
Soirée bénéfice pour ceux qui ne seront pas là en l'an deux mille
}

Fragments d'une pièce inédite

\section{Michel Marc Bouchard}

Volume 33, numéro 1 (97), automne 2007

Michel Marc Bouchard

URI : https://id.erudit.org/iderudit/017526ar

DOI : https://doi.org/10.7202/017526ar

Aller au sommaire du numéro

\section{Éditeur(s)}

Université du Québec à Montréal

\section{ISSN}

0318-9201 (imprimé)

1705-933X (numérique)

Découvrir la revue

Citer ce document

Bouchard, M. M. (2007). Soirée bénéfice pour ceux qui ne seront pas là en l'an deux mille : fragments d'une pièce inédite. Voix et Images, 33(1), 31-45.

https://doi.org/10.7202/017526ar d'utilisation que vous pouvez consulter en ligne.

https://apropos.erudit.org/fr/usagers/politique-dutilisation/ 


\author{
SOIRÉE BÉNÉFICE POUR CEUX \\ QU I NE SERONT PAS LÀ EN L'AN DEUX MILLE \\ Fragments d'une pièce inédite \\ $+++$
}

MICHEL MARC BOUCHARD

Dans toutes les transcriptions, les passages biffés sont signalés par le texte barré. Les ajouts sont en gras et sont placés au-dessus ou en dessous du texte afin d'indiquer leur emplacement dans le tapuscrit. Étant donné le nombre de versions et le difficile travail de correction que l'auteur évoque dans l'entretien, il nous a semblé intéressant d'indiquer ces passages, même s'ils ne sont pas très nombreux.

Le premier document reproduit des extraits tirés des trois premières versions de la pièce; le second est une transcription des scènes 1 à 5 de la quatrième. Le document a visiblement servi à une mise en scène, car il porte des indications de son, de déplacements d'acteurs et d'éclairage. Toutefois, dans la transcription, nous n'avons reproduit que les marques faites de la main de l'auteur.

VERSION I

\title{
PREMIÈRE PARTIE
}

\section{PRÉFACE}

\section{VOIX-OFF DE LILI}

Je le déclare à quiconque entend les paroles de la prophétie de ce livre: si quelqu'un y ajoute ou y retranche quelque chose, il sera frappé des fléaux décrits dans ce livre. Lili Mercier, 14 ans. Janvier 1990.

LA MISE À MORT DE DIEU dédiée à tous ceux qui ne seront pas là en l'an 2000. Premier verset. Il y avait, ce soir-là, une pluie d'étoiles filantes d'une beauté exceptionnelle. Mathilde, la vieille louve, trônaitera sur la cage. Elle fera un vœu différent à chaque étoile, tout en écoutant Flora, la dernière fleur d'Amazonie, jouer du violoncelle... Martin, l'Ange gardien, sera agenouillé, contemplant lui aussi les étoiles. Ces mêmes étoiles filantes qui l'avaient transporté sur la terre afin qu'il guide la destinée du dernier enfant de la Gouve... Il suppliera son Dieu de retarder l'échéance... Ce sera une route du nord. Ce sera l'hiver. Ce sera deux heures avant l'arrivée de l'an 2000. 
Premier verset. L'Ange doutera!

(Le décor apparaît. Mathilde enveloppée dans une fourrure de loup est assise sur une voiture des années quatre-vingt. Elle écoute Flora jouer du violoncelle. Martin est à genoux.)

\section{VERSION II}

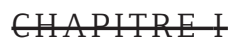

(Un livre sur un bureau. Une chaise.)

\section{VOIX-OFF DE MARTIN}

Lili, faut que tu te changes. J'ai choisi la robe noire. T'as une préférence pour les bijoux? (Silence.) Ton père veut que tu sois belle pour le jour de l'an. Y'reste rien que deux heures avant l'an 2000. Viens ici, j'vas te coiffer. Ta grand-mère arrive de voyage à soir. A va certainement te donner un beau cadeau. Lili, arrête d'écrire un peu, pis viens te faire peigner.

MARTIN (entrant avec la brosse à cheveux.)

Lili? J'aime pas ça me parler tout seul? (Il voit le livre.) Que c'est qu'une fille sage comme elle peut ben écrire? (Appelant.) Lili? (Il s'installe au bureau.) J'ai pas l'droit de faire ça. (Appelant.) Lili? (Il ouvre la première page.) Après toute, c'est moi qui m'occupe d'elle. Ça fait partie de mon travail.

MARTIN (Lisant.)

POUR TOUS CEUX QUI NE SERONT PAS LĀ EN L'AN 2000, de Lili Mercier.

- Drôle de titre.

\section{CHAPITRE I}

\section{VOIX-OFF DE MARTIN}

C'était une vieille voiture des années quatre-vingt sur une route du nord. Paysage d'hiver. Mathilde, la grand-mère, était emmitouflée dans une fourrure de loup, et assise sur le capot. Elle écoutait Flora, la Brésilienne, jouer du violoncelle, assise sur une valise. Martin avait la tête dans les étoiles. Sept heures avant l'arrivée de l'an 2000.

(Le décor apparaît.)

VERSION III

Décor: une voiture des années quatre-vingt. Une route du nord. Hiver. 


\section{LILI}

Premier verset. Ce soir-là, il y aura une pluie d'étoiles filantes d'une beauté exceptionnelle. Mathilde, la vieille louve, trônera sur la cage! Elle fera un vœu différent à chaque étoile, tout en écoutant Flora, la dernière fleur d'Amazonie, jouer du violoncelle... Bruno, le loup de paille, demandera au ciel d'apaiser son courroux. PierreLouis, le loup de sang, restera à l'intérieur de la cage et Martin, le loup d'argent, observera la scène. Nathalie sera là, pas la vraie, la créature de l'enfant. Le temps sera venu de la révolte des enfants. Ce sera une route du nord. Ce sera l'hiver. Ce sera... deux heures avant l'arrivée de l'an 2000.

(Lili entre dans la voiture.)

(Mathilde, enveloppée dans une fourrure de loup, est assisse sur le capot de la voiture. Martin tourne les feuilles de musique pour Flora qui joue du violoncelle. Pierre-Louis et Nathalie sont assis dans la voiture.)

\section{VERSION IV}

\section{PREMIẼRE PARTIE}

\section{SCÈNE 1}

(Flora joue du violoncelle.)

(Pierre-Louis est blotti dans les bras de Mathilde. Elle porte une fourrure de loup. Bruno écoute la télévision.)

\section{VOIX OFF DU TÉLÉVISEUR}

Ce soir, le grand administrateur et sa dame étaient les invités du célèbre défilé annuel des Fourrures Tanguay. L'absence de madame Mathilde Tanguay a créé tout un émoi... On est sans nouvelles de l'excentrique dame de la fourrure depuis dix-huit heures ce soir... Les participants de cette soirée bénéfice dont les fonds iront aux victimes de la crise économique s'expliquent mal cette disparition. Le grand administrateur a demandé à la police et à l'armée d'engager tous les efforts afin de retrouver madame Mathilde...

\section{BRUNO}

C'est la deuxième fois qu'y parle de vous.

PIERRE-LOUIS

On pourrait se passer de la T.V. icitte!

\section{BRUNO}

Quoi? 


\section{PIERRE-LOUIS}

Ferme la T.V.! (Bruno ferme la télévision.) Que c'est qu'y attendent pour que ça marche l'effet de serre? On gèle! C't'une bonne idée que t'as eu Bruno de prendre un raccourci pour être là avant tout le monde!

\section{BRUNO}

Y'a certainement une auto qui va finir par passer.

\section{PIERRE-LOUIS}

Une route du nord, le soir du jour de l'an, faut pas s'attendre à un embouteillage.

\section{BRUNO}

Les services de déneigement vont passer. C'est pas de l'asphalte chauffante ici.

\section{PIERRE-LOUIS}

Faudrait d'abord qui neige. C't'idée de vivre aussi loin de la civilisation!

\section{BRUNO}

On est chanceux que personne se soit fait mal.

PIERRE-LOUIS

On est ben chanceux! Je me demandais depuis quecques années comment j'allais passer mon jour de l'an de l'an deux mille. Je m'étais dit que ça allait être une soirée spéciale. On est mardeux comme ça se peut pas!

(Cessant de jouer.)

FLORA

Minhas manos estos frias!

(Temps.)

PIERRE-LOUIS (À Mathilde)

Tantôt quand on a frappé l'orignal, j'ai pensé que je pouvais te perdre.

\section{FLORA (Cessante jout)}

Minhas manos estos frias!

PIERRE-LOUIS

J'ai pas pensé à moi, j'ai pensé rien qu'à toi.

FLORA

Minhas manos estos frias. 


\section{PIERRE-LOUIS}

Cout'donc, on est pas capable d'être tranquille deux secondes sans que le TiersMonde nous rappelle qu'y existe?

\section{BRUNO}

Allez vous réchauffer dans la voiture Flora.

PIERRE-LOUIS

Joue encore, Flora!

BRUNO

T'aimes la musique, ast'heure?

PIERRE-LOUIS

Ça me détend! T'as pas remarqué!

BRUNO

Elle gèle des mains!

\section{PIERRE-LOUIS}

Moi aussi! Je te ferais remarquer que tu t'es pas encore excusé d'avoir saboté notre belle soirée bénéfice. T'as dû t'excuser à tous les saints du ciel, mais nous autres on doit pas être assez dignes.

\section{BRUNO}

Dis pas ça!

\section{PIERRE-LOUIS}

Y va descendre une grosse paire de ciseaux des nuages pour me couper la langue?

\section{BRUNO}

C'est pas le temps de se moquer de ça.

\section{PIERRE-LOUIS}

Je sais pas de qui on se moque le plus présentement? Je les entends rire d'ici nos cinq cents invités! J'les entends remplir leux verres de farces plattes! Ça doit être beau comme défilé. Quarante mannequins laissés à eux-mêmes... Quarante mannequins qui vont porter les quatre cents manteaux que j'ai dessinés comme s'ils portaient des poches de patates... La collection des fourrures Tanguay... La collection du millénaire! Pis oùsqu'elle est la famille Tanguay? Oùsqu'y sont ceux-là qui ont organisé une belle soirée bénéfice, un beau réveillon pour donner de l'argent aux pauvres pis aux handicapés? Oùsqu'y sont ceux-là qui ont poussé le mauvais goût jusqu'à présenter la fine pointe de la mode pour financer les horreurs du genre humain? Perdus! Perdus dans le bois à moins dix sous zéro parce que le plus attardé 
de la famille a décidé d'aller à chasse avec son char. Envoye, prie-le ton bon Dieu. Remercie-le que je t’aye pas encore égorgé.

\section{BRUNO}

C'est un accident.

PIERRE-LOUIS

J'ai toujours su que t'étais rien qu'un accident.

\section{MATHILDE}

O.K. ! Pierre-Louis. C'est assez!

PIERRE-LOUIS

Depuis quand tu prends sa défense?

\section{MATHILDE}

Tu vas avoir mal à tes greffes!

PIERRE-LOUIS

Si y'a quelqu'un qui sait si ses greffes y font mal, c'est moi. J'ai pas besoin de toi pour ça.

\section{SCÈNE 2}

(ELLE sort de la voiture avec un récipient en main. Elle porte un manteau de laine.)

\section{ELLE}

Continuez à vous engueuler! Dérangez-vous pas pour moi!

\section{MATHILDE}

Oùsque tu vas?

ELLE

J'vais chercher de l'eau.

PIERRE-LOUIS

Tout est gelé!

ELLE

Y'a une source qui coule pas loin.

(Elle sort.) 


\section{SCÈNE 3}

\section{MATHILDE}

J'ai rencontré vot'père par une belle grosse lune d'hiver pareille à celle d'à soir. C'était dans le temps oùsque je m'occupais moi-même de mes pièges. C'est un soir oùsque la brunante est tombée plus de bonne heure que de coutume. Je finissais de faire la tournée de mes pièges quand je me suis aperçue que la neige pis le vent avaient effacé la piste, effacé mes traces, comme si j'avais jamais passé par là, comme si j'avais jamais existé. J'ai paniqué. J'ai appelé au secours. Plusieurs fois. Les bourrasques de vent faisaient tourbillonner mes appels de détresse au-dessus de ma tête, sans jamais se rendre plus loin. Le froid s'infiltrait sous mes vêtements... J'avais envie de dormir du sommeil du froid, celui dont personne se réveille. Avant de sombrer, j'ai vu sa silhouette au travers de la poudrerie. Triomphal devant moi... Majestueux avec la grosse lune qui l'enveloppait... Ses yeux brillaient dans l'obscurité...

\section{PIERRE-LOUIS}

Pourquoi tu nous racontes ça?

\section{MATHILDE}

La lune bouge plus depuis l'accident. J'ai l'impression que le temps s'est encore arrêté.

\section{PIERRE-LOUIS}

On a pas connu not'père pis ça nous a jamais manqué!

\section{MATHILDE}

Y s'est approché de moi, tellement proche que je pouvais le toucher. Son museau frôlait mes vêtements. J'ai compris que je devais le suivre. Au lieu de me ramener vers le camp des trappeurs, y m'a guidée à son liteau... Le lendemain matin, la tempête faisait encore rage. J'ai quand même décidé de retourner au camp, y fallait que je retourne dans mon monde... Je l'ai vu me suivre à distance. J'ai vu dans ses yeux qu'y me demandait de rester. J'ai entendu un coup de feu... un autre... un troisième pis j'ai vu le loup, vot'père, s'écrouler. La tempête s'est évanouie au moment oùsque j'y ai fermé les yeux. Le vieil Esquimau m'a fait face avec son fusil...

\section{PIERRE-LOUIS}

Un Inuit!

\section{MATHILDE}

Y s'est mis à me baragouiner quecque chose dans sa langue...

PIERRE-LOUIS

Esquimau, c't'un vieux nom. 


\section{MATHILDE}

La lune faisait face au soleil, la lune qui s'était arrêtée de bouger durant la nuit, est retournée se coucher.

PIERRE-LOUIS

Un Inuit!

MATHILDE

J'y changerai pas de race pour te faire plaisir.

PIERRE-LOUIS

Un Inuit! C'était un Inuit!

MATHILDE

Un Inuit, calvaire!

BRUNO

On sacre pas!

MATHILDE

Dans mon monde, c'est pas la place pour une belle instruction, c'est pas nécessaire de savoir que les Esquimaux s'appellent plus des Esquimaux... C'est la place pour le bois, le trappage pis l'argent!

PIERRE-LOUIS

Un Inuit!

(On entend, au loin, un hurlement de loup. Long silence.)

MATHILDE

C'est Martin? Êtes-vous capable de me dire c'qu'y fait ?

BRUNO

Le vent est pas du bon côté!

MATHILDE

Ça fait longtemps qu'y est parti prendre sa marche.

BRUNO

Une bonne heure. Depuis l'accident.

MATHILDE

Martin a plus l'habitude du bois. 


\section{PIERRE-LOUIS}

Gêne-toi pas pour t'inquiéter de lui devant moi.

MATHILDE

Depuis qu'y est revenu, y'a rien dit!

PIERRE-LOUIS

L'enfant prodigue que tu payes pour qu'y revienne nous voir après des années!

\section{MATHILDE}

Même quand on a eu l'accident tantôt, y'a rien dit!

\section{PIERRE-LOUIS}

Martin! Martin! Martin! Le beau Martin qui te chierait dessus pis tu dirais : encore! Penses-tu que je sais pas pourquoi tu l'as fait revenir? Penses-tu que je sens pas tes chaleurs de vieille femme?

\section{MATHILDE}

Bruno, apporte ses calmants!

\section{PIERRE-LOUIS}

Trouve-moi une raison pour laquelle je devrais me calmer. Trouves-en une, toi qui es tellement...

\section{MATHILDE}

$\mathrm{T}^{\prime}$ oserais insulter la vieille femme en chaleur une deuxième fois?

FLORA

Eu estou completamente gelata!

PIERRE-LOUIS (Retournant sa rage sur Flora.)

Y fait frette dans not'pays, t'as rien qu'à t'adapter. Tout c'qu'elle a à faire c'est de jouer d'un petit peu de musique pis elle réussit à se plaindre?

\section{MATHILDE}

Apporte
Ap pilules blanches, Bruno!

\section{PIERRE-LOUIS}

Pis c'est c'te coquerelle-là qu'on me greffe, pièce par pièce, pour que je survive. J'ai déjà sa hanche, l'épiderme de mon bras, la peau de sa cuisse. Qu'elle se gèle les mains, comme ça je serais sûr de pas les avoir. Pour vous autres, le temps, c'est pas compliqué. Vous pouvez vous payer le luxe d'un accident pis d'attendre calmement les secours. Moi, c'est mon dernier défilé! C'est mon dernier défilé... C'est mon 
dernier jour de l'an! Pis tu t'inquiètes de Martin! Tu pourrais avoir la décence $\mathrm{d}$ 'attendre que je soye mort avant de me remplacer!

Joue, Flora! Le silence de l'hiver, c'est le silence de la mort! Joue jusqu'à demain matin, jusqu'à ce que le soleil vienne encore m'arracher la peau! (On entend un autre hurlement de loup au loin.) C'est pas juste! C'est pas juste!

\section{SCÈNE 4}

\section{BRUNO}

Vous étiez pas obligée d'exciter Pierre-Louis en y parlant de Martin!

\section{MATHILDE}

J'ai failli passer toute entière par le pare-brise de l'auto, grâce à toi; nos fourrures défilent sans nous autres, grâce à toi; j'me passerais de tes commentaires, ciboire!

\section{BRUNO}

On sacre pas!

\section{MATHILDE}

C'est ça! On sacre pas, on crache pas, on pète pas. On garde tout ça par en dedans, c'est plus moderne.

\section{BRUNO}

On sacre pas!

\section{MATHILDE}

À soir, j'avais réussi à ramasser tout c'qui reste de présentable, de respectable, tous ceux qui pensent encore qu'un manteau de fourrure sur l'dos, ça veut dire quecque chose. Le Grand Administrateur pis la première dame... des mois de lichages de fonctionnaires pour qu'y soient présents. Des acheteurs du monde entier pendus à leux téléphone... Les plus beaux mannequins; pas une jambe en bas de six pieds, pas une lèvre en bas de trois pouces. Des femmes avec des poitrines en i pis des gars avec des poitrines en $u$. Des beaux corps avec des beaux cheveux pour que tout l'monde se pense beau comme eux autres si y'achètent nos fourrures. J'ai payé des bras pour casser les gueules des écologistes, au cas... J'ai supplié des mognons d'handicapés en chaises roulantes pour faire pitié devant les écologistes, au cas... J'avais préparé chaque seconde de mon apparition à la fin du défilé; La Grande Dame de la fourrure avec ses enfants! Même Martin était là! Veux-tu ben me dire pourquoi t’as pas évité l'orignal? Pourquoi t’as pas freiné? Pourquoi t’as accélérée? Pourquoi t'as pas apporté le téléphone? Tu voulais être sûr que personne vienne à notre secours? Je t'ai vu lâcher le volant!

\section{me}

Pourquoi t'as voulu tuer? (Temps.) T'étais trop lâche pour nous diriger sur un arbre? Y'a fallu qu'un animal se mette sur not'chemin? Arrête de nettoyer l'auto quand je te parle! 


\section{BRUNO}

Vous aimez ça voir le sang, les morceaux de chair?

MATHILDE

Vous êtes nés dans l'sang, je vous ai élevés dans le sang, c'est pas quecques gouttes de plus ou de moins qu'y vont faire la différence.

BRUNO (Quittant son travail.)

Pis là, qu'est-ce que je fais?

\section{MATHILDE}

T'aimerais ça que je t'ouvre les bras pour te pardonner? Mauvais fils! (Elle le gifle.) Excuse-toi!

\section{BRUNO}

J'ai l'impression que vous m'avez giflé mais c'est juste une impression. Le genre d'impression qu'on a lorsqu'on assiste à un événement tragique à la télévision; l'impression que ça nous fait mal, mais c'est juste une impression. L'impression que je vous parle pis que vous m'entendez jamais! L'impression d'une lune qui bouge plus. L'impression d'un apocalypse qui s'en vient. Dans l'auto, j'voyais le paysage défiler comme dans un corridor sans fin. Des arbres de chaque côté-les l... C'est juste une impression de forêt, c'est juste une façade; y'a cent pieds d'arbres de chaque côté, pis après on a tout rasé. t'au.. Des branches d'arbres morts en émergent. Des bras de noyés appelant Un corridor sans fin. Nous autres dans l'auto, silencieux comme dans un tombeau... Nous autres qui trouvent jamais rien à se dire, si c'est que pour se mordre. Pis y'a eu la ptite tempête, soudaine, pis de l'aut'côté de la poudrerie, j'ai vu les yeux sur la route, des yeux rouges qui fonçaient sur la voiture. J'ai lâché le volant! Je voulais que tout arrête. J'avais besoin que le temps s'arrête. Je voulais qu'on se parle pour une fois. Peut-être la dernière, mais pour une fois! Qu'est-ce qu'y vous a dit le vieil Esquimau avec le fusil?

\section{MATHILDE}

Excuse-toi !

BRUNO

J'm'excuse!

MATHILDE

J'ai pas compris c'qu'y m'a dit. Y parlait inuit!

\section{BRUNO}

Vous voulez pas qu'on se parle? 


\section{MATHILDE}

Tu vas aller dire à nos invités que la Grande Dame de la fourrure a eu un accident! Tu vas aussi leux dire qu'on va leux faire des bons prix pour se faire pardonner.

\section{BRUNO}

J'en ai pour des jours à marcher.

\section{MATHILDE}

Tu courras.

\section{BRUNO}

Vous avez vu les étoiles?

\section{MATHILDE}

C'est une pluie d'étoiles filantes. Fais des vœux pis arrête de te conter des peurs.

\section{BRUNO}

Elles se décrochent du ciel, l'une après l'autre, pour tomber quelque part sua planète. Sans bruit! Ça ressemble à un feu d'artifice au-dessus d'une ville bombardée. Comme à la télévision. Si elles venaient à toutes disparaître, ce serait la grande noirceur. J'aime pas la noirceur.

\section{MATHILDE}

Tu peux tout voir dans le noir. T'as les yeux de ton père.

\section{BRUNO}

À soir, les églises sont pleines de monde!

\section{MATHILDE}

Tant mieux pour eux autres; la quête va être bonne! J't'aimais mieux quand tu prenais de la coke! Depuis que tu sniffes du Bon Dieu, t'es plus parlable. Ma génération a essayé d'oublier ton Bon Dieu. Mais y revient! Toujours plus fanatique, avec une nouvelle face, à chaque fois. Y'a trente ans, y fumait du pot pis y faisait l'amour avec tout l'monde, ast'heure y administre la fin du monde pis y mange biologique. Ma religion, c'est la finance. Mes dévotions, les taux de change pis l'indice boursier; pis mon apocalypse, c'est la faillite.

\section{BRUNO}

C'est horrible de parler comme ça.

\section{MATHILDE}

Horrible! C'est un vieux mot, ça aussi. Ça ne veut plus rien dire. Les enfants de la famine, les charniers de la guerre, les tragédies familiales, on lit tout ça, calmement, en sirotant not'café. Sais-tu que je suis en train de philosopher pis t'es toujours pas parti? Flora, jouez! 


\section{BRUNO}

Elle a les mains congelées!

MATHILDE

Jouez! Quand Bruno sera parti, vous irez vous réchauffer.

(Flora se met à jouer. Péniblement.)

BRUNO

Vous avez aucune pitié!

MATHILDE

La pitié, c'est la monnaie des pauvres!

BRUNO

Pis encore moins de conscience!

\section{MATHILDE}

Sais-tu combien Fora a chargé pour la peau de sa cuisse? Le Tiers-Monde pis moi, on a la même conscience. On parle, pis on parle pis t'es toujours pas parti?

\section{BRUNO}

C'est peut-être la dernière fois qu'on se voit...

\section{MATHILDE}

J'ai enfanté Martin pis ta sœur, la race qui fait manger les loups dans leux mains; j'ai enfanté Pierre-Louis, la race qui se fait mordre parce que sa senteur inspire pas confiance. Pis ta race; celle qui reste de l'aut'côté de la clôture avec un rond de pisse dans ses culottes! Tu réussiras pas à me faire peur. La pisse de la peur, c'est ta spécialité, pas la mienne.

BRUNO

Détrompez-vous, maman, c'est pas de la peur...

MATHILDE

J'te défends de m'appeler comme ça!

BRUNO

Si Papa était là avec nous autres...

MATHILDE (Caressant son manteau.)

Y'est là, avec nous autres! 


\section{BRUNO}

Si y avait su parler... Y vous aurait dit si tu savais comme je t'aime! Par les temps qui courent... dans mes bras vous allez pouvoir vous apaiser.

MATHILDE

Flora, j'ai manqué quecques notes! Recommencez!

BRUNO

Maman!

MATHILDE

Si le ridicule tuait, ça ferait longtemps qu'on pleurerait sur ta tombe!

BRUNO

Qu'est-ce qui vous a dit le vieil Esquimau avec son fusil?

MATHILDE

J'sais pas pourquoi j'vous ai raconté ça!

FLORA (Cessant de jouer)

Minhas manos estos frias!

BRUNO

En chemin, j'vais prier pour nous autres.

MATHILDE

Si jamais y te répond, dis-y d'aller nicher ailleurs que dans ma meute.

(Bruno sort.)

\section{SCÈNE 5}

\section{MATHILDE}

Vous avez vu Flora? J'ai élevé du carcajou, du rat musqué, du renard argenté, du renard roux, du castor, de la loutre. J'ai élevé du lynx, du vison de terre, du vison de mer, du chat sauvage, de l'hermine, du cougar de l'est, de la martre, de la belette. Pis j'ai élevé mes quatre enfants.

\section{FLORA (Cessant de jour)}

O que voce esta dizendo, Mathilde?

\section{MATHILDE}

Tous les quatre, le même jour, la même portée. 


\section{FLORA}

Eu quero ir para o caro.

\section{MATHILDE}

Tout ce que j'ai dû faire pour garder le contrôle. J'ai appris à fonctionner comme euxautres. J'ai appris leux lois. J'ai appris à les dompter, à trouver leux faiblesses... À soir, j'ai peur d'avoir peur.

FLORA

Mathilde!

MATHILDE (Caressant son manteau.)

Quand j'ai eu fermé les yeux du loup, le vieil Esquimau m’a fait face avec son fusil. Même si je ne comprenais pas sa langue, j'ai compris... On ne couche pas avec le loup. Tu portes dans ton sein la progéniture de ton crime. Tu portes la vengeance des astres; des enfants mi-hommes, mi-loups, des enfants qui vont être immortels, des enfants qui se vengeront du destin que tu leux a donné. On couche pas avec le loup. J'ai su toute ma vie qu'un moment comme à soir se devait d'arriver. J'ai peur, Flora! J'ai peur que mes enfants sentent ma peur!

FLORA

Pelo amor de deus.

MATHILDE

Allez mettre vos mains à chaleur. On en aura peut-être pas besoin mais, au cas... 